\title{
Effects of ingredients on the characteristics of rice cakes
}

\begin{abstract}
Steamed leavened rice cakes made from rice flour, sugar, water, yeast or baking powder were used to study the effects of ingredients on cereal-based cakes. Volume expansion linearly correlates to amylopectin and negatively correlates to amylose content ( $2=0.84$ ), with an optimum amylose/amylopectin ratio of approximately $1: 8$. Higher amylopectin content resulted in sticky, elastic and unacceptable cake. The optimum water content for volume expansion was in the range of $90 \pm 5 \%$ by weight of rice flour. Sugar content correlates linearly with volume expansion and tenderness of chemically leavened rice cakes (CLRC) ( $\mathrm{r}$ $2=0.99$ and 0.92 , respectively). Sugar content above $50 \%$ of rice flour weight decreased volume and softness of yeast-leavened rice cakes (YLRC). Tenderness of YLRC correlates linearly to fat content ( $\mathrm{r} 2=0.98$ ), but volume expansions correlates negatively to the square root of fat content ( $\mathrm{r} 2=0.81$ and 0.97 for the two rice cakes). Both tenderness ( $2>0.92$ ) and volume expansion ( $2>0.93$ ) correlates linearly with emulsifier content up to $3 \%$ by weight of rice flour, above which the cake becomes crumbly. A little salt increased hardness, but further increase reduced both volume expansion and hardness of rice cakes. Skimmed milk powder greatly increased the expansion of CLRC. Ovalbumin content correlates linearly to volume expansion of YLRC ( $2=0.87$ ) but negatively to the expansion of CLRC ( $\mathrm{r} 2=$ 0.96). The proteins that improved the textures of rice cakes were skimmed milk and egg white with an optimum content of 2-4\%. Egg yolk, soya protein, whey powder and wheat gluten reduced both tenderness and volume expansion. The effects of the various ingredients interacting together were studied and the optimum formulations was determined using a Random Centroid Optimisation Program.
\end{abstract}

Keyword: Amylose/amylopectin; Egg; Emulsifier; Expansion; Fat; Gluten; Milk; Protein; Rice cakes (apam); Salt; Soya; Sugar; Texture; Whey 